\title{
ESTUDIO DE EVENTOS FENOLÓGICOS EN Cratylia argentea (Desv.) $O$ Kuntze CULTIVAR VERANIEGA
}

\author{
María Mesén Villalobos' ${ }^{1}$, William Sánchez Ledezma ${ }^{1}$
}

\begin{abstract}
RESUMEN
Cratylia argentea es una leguminosa forrajera arbustiva originaria de América del Sur. El objetivo del presente estudio fue realizar el seguimiento de los principales eventos fenológicos durante siete años, con el propósito de contribuir al conocimiento y a la propagación del cultivo en Costa Rica. El trabajo se desarrolló en una finca ubicada en el caserío El Estero, distrito San Antonio, cantón Puriscal, provincia San José. La topografía de la finca es irregular, la altitud $1.045 \mathrm{msnm}$ y la temperatura y precipitación promedio anual son de aproximadamente $21,00{ }^{\circ} \mathrm{C}$ y $2.541 \mathrm{~mm}$, respectivamente. La siembra se hizo de forma directa durante el mes de setiembre de 1998, a un metro entre surcos y entre golpes de siembra. La emergencia de plantas ocurrió a los 10 días. La floración se presentó durante el mes de setiembre, un año después de la siembra. En el transcurso de los años siguientes también hubo floración en el mes de setiembre, pero se prolongó hasta abril. Las últimas flores no se desarrollaron ya que el inicio de las lluvias provocó exceso de humedad, lo que ocasiona deterioro y caída de los botones florales. La cosecha se realizó de febrero a abril, diariamente para evitar pérdidas por dehiscencia. La producción de semilla fue de $0,12 \mathrm{t} /$ ha durante el primer año, ascendiendo a 2,45 t/ ha para el cuarto año; descendiendo a $0,83 \mathrm{t} /$ ha durante el sétimo año. El tamaño promedio de las vainas fue de $13,80 \mathrm{~cm}$ y la cantidad de semillas por vaina fue de 7,10 unidades. La cantidad promedio de semillas por kilogramo fue de 3.650, con una germinación que osciló entre 85 y $90 \%$. Se concluye que Cratylia argentea tiene un desarrollo inicial lento, una floración desuniforme y una producción de semilla con un comportamiento de forma parabólica durante el periodo de evaluación.
\end{abstract}

Palabras clave: Cratylia argentea, cultivar Veraniega, eventos fenológicos, arbusto, forraje.

\section{INTRODUCCIÓN}

La ganadería bovina de carne y doble propósito en áreas tropicales depende en gran medida de los forrajes principalmente gramíneas, los cuáles generalmente no satisfacen los requerimientos de los animales. En general, los rumiantes que consumen pastos de baja calidad sufren deficiencias principalmente de energía, proteína y minerales.

En algunos casos los sistemas intensivos, a base de gramíneas fertilizadas, son una alternativa para mejorar la producción animal, sin embargo, cada vez es mayor la necesidad de encontrar sistemas de bajos insumos para lograr una producción animal eficiente y en armonía con la naturaleza. Entre las opciones de alimentación que utilizan prácticas de uso de la tierra en forma sostenible se incluyen: los bancos de proteína, la siembra de cultivos forrajeros adaptados a suelos pobres, el uso de asociaciones herbáceas y arbóreas y muchos otros más (Martínez 1992).

En este sentido, las leguminosas en monocultivo o en asocio con gramíneas han sido reconocidas como fuente de

${ }^{1}$ INTA (Instituto Nacional de Innovación y Transferencia en Tecnología Agropecuaria) Costa Rica. 
excelente forraje y como mejoradoras de la fertilidad del suelo. Su habilidad para fijar nitrógeno del aire y su alto contenido de proteína y minerales las hacen indispensables en la alimentación animal (Bernal 1991).

Un arbusto que ha dado muy buenos resultados en este sentido es Cratylia argentea que es originario de América del Sur, el cual ha sido evaluado y seleccionado por su buena adaptación a zonas con sequías prolongadas y suelos ácidos de baja fertilidad. (Argel y Lascano 1998 consultados por Argel et al. 2001, Orozco 2003 y Orozco 2005).

El género Cratylia pertenece a la familia Leguminoseae, subfamilia Papilionoideae, tribu Phaseoleae y subtribu Diocleinae (Lascano et al. 2002). Esta leguminosa que alcanza entre 1,50 y 3,00 metros de altura, florece y produce semilla de buena calidad en condiciones de trópico húmedo o subhúmedo. Fue introducida a Costa Rica en el año 1988 y liberada como cultivar Veraniega en el año 2001 (Argel et al. 2001).

El cultivar Veraniega es una mezcla física de las accesiones Cratylia argentea CIAT 18516 y Cratylia argentea CIAT 18668, la cual se obtuvo al combinar cantidades iguales de semilla de estas dos accesiones y utilizar dicha semilla para experimentación. Lo mencionado anteriormente se llevó a cabo porque existía información procedente de México, Brasil, Colombia, Panamá y Costa Rica que demostraba que esas dos accesiones evaluadas eran las que mostraban mejores atributos y muy similares entre sí (Argel 2008) ${ }^{2}$.

En Costa Rica esta leguminosa se ha propagado por medio de semilla sexual, en sitios que van desde 0 hasta $1.045 \mathrm{msnm}$ la propagación por estaca no es efectiva.
Durante el año 1998 se introdujo Cratylia argentea a la Región Pacífico Central de Costa Rica a través del Proyecto de Reforestación de Fincas Ganaderas para realizar sus respectivas evaluaciones, con el fin de buscar mejores opciones de alimentación para la ganadería de la región.

El objetivo del presente estudio fue realizar el seguimiento de los principales eventos periódicos naturales involucrados con el arbusto cuyo propósito fue contribuir al conocimiento fenológico y a la propagación del cultivo en Costa Rica.

\section{MATERIALES Y MÉTODOS}

El presente trabajo se desarrolló en una finca ubicada en el caserío El Estero, distrito San Antonio, cantón Puriscal, provincia San José. La topografía es irregular, situada a una altitud de $1.045 \mathrm{msnm}$, con una temperatura y precipitación promedio anual de aproximadamente $21^{\circ} \mathrm{C}$ y $2.541 \mathrm{~mm}$, respectivamente.

La siembra de $250 \mathrm{~m}^{2}$ de área se realizó el 17 de setiembre de 1998, y se utilizó la técnica de cero labranza. Se empleó únicamente herbicida sistémico no selectivo, sembrando directamente en el suelo a una distancia de un metro entre surcos y un metro entre plantas depositando 3 semillas por golpe de macana. La profundidad de siembra fue de $1,50 \mathrm{~cm}$, con el terreno suelto, dando doble golpe de macana en forma cruzada. La semilla se inoculó previamente con la cepa CIAT 3565 a razón de $50 \mathrm{~g}$ por $\mathrm{kg}$ de semilla. El inóculo se adicionó a la semilla diluido en agua de azúcar al $1,50 \%\left(\right.$ Uribe 1998) ${ }^{3}$.

Antes de la siembra se realizó el análisis de suelo, (Cuadro 1). La primera fertilización

2Argel, P. 2008. Origen del cultivar Veraniega (correo electrónico). Colombia. CORPOICA. Comunicación personal.
3Uribe, L. 1998. Inoculación de Cratylia argentea (consulta). San José, CR, Universidad de Costa Rica. Comunicación personal. 
se hizo a los 234 días de crecimiento de las plantas, a razón de $15 \mathrm{~g}$ de triple superfosfato (46 \% de $\mathrm{P}_{2} \mathrm{O}_{5}$ ) por planta. Esta misma cantidad se aplicó durante cada uno de los años siguientes al inicio de las lluvias (Argel 1998) ${ }^{4}$. Cada año después de la cosecha, los arbustos se podaron y aproximadamente un mes después, se realizó la aplicación de fertilizante coincidiendo con el inicio de las lluvias. Estas fueron las únicas prácticas de manejo del cultivo realizadas durante los años de producción evaluados.

Se dio seguimiento a los eventos fenológicos durante la fase de establecimiento y luego durante los siete años consecutivos de producción. Los principales eventos registrados fueron los siguientes:

\section{Tasa de crecimiento}

Se tomó en cuenta la emergencia de las plantas para luego medir la altura de las mismas cada semana durante la fase de establecimiento y desarrollo hasta la primera poda. Luego se determinó la tasa de crecimiento durante los primeros 60 días y durante el periodo completo de la siembra a la primera poda.

\section{Días a floración}

Durante la fase de establecimiento y desarrollo se anotaron los días transcurridos de la siembra a la floración, considerando la floración cuando el $50 \%$ de las plantas tuvieron alguna flor. Del segundo año en adelante, se anotó el mes de máxima floración, ya que la misma permaneció durante todo el período de cosecha.

\section{Días a fructificación}

Durante la fase de establecimiento y desarrollo se anotaron los días transcurridos de la siembra al inicio de la cosecha, la cual se inició al existir cambio de color en las vainas. Del segundo año en adelante se anotó el tiempo que permaneció la cosecha.

\section{Producción de semilla}

Para evaluar la producción de semilla se utilizó el método de recolección total de las vainas en toda la plantación. Se cosecharon las vainas directamente del arbusto, cuando cambiaban de color verde a café claro. El material cosechado se mantuvo al sol y cuando la mayoría de las vainas abrieron se terminó de extraer las semillas manualmente ya que muchas quedaron adheridas a las valvas. Por último, la semilla pura se separó de la basura por medio de una zaranda, la cual separó materiales extraños y residuos de vainas. Después de realizar este procedimiento, la semilla limpia se secó al sol un día más, para luego proceder a su almacenamiento. La producción total se pesó y se calculó la producción por hectárea. Este procedimiento se repitió durante siete cosechas consecutivas.

Con los datos de la producción de semilla colectada durante cada uno de los siete años (Cuadro 4) se realizó un análisis de regresión. Se utilizó el software de estadística y biometría Info Stat 2001 (UNC 2001).

\section{Porcentaje de germinación}

Se determinó el porcentaje de germinación de la semilla durante cada año de estudio para luego reportar el rango en que osciló el parámetro. La prueba se hizo en laboratorio a temperatura ambiente en recipientes de cristal con tapa, con papel filtro y humedad necesaria para estimular la germinación.

\section{RESULTADOS Y DISCUSIÓN}

El análisis de suelo (Cuadro 1) presentó un valor bajo de $\mathrm{pH}$ y alto en aluminio, como consecuencia el porcentaje de acidez es alto $22,80 \%$. Los contenidos de $\mathrm{Ca}, \mathrm{Mg}, \mathrm{K}$, $\mathrm{P}, \mathrm{Mn}, \mathrm{Cu}$ y las relaciones $\mathrm{Ca} / \mathrm{Mg}$ y $\mathrm{Mg} / \mathrm{K}$, estaban en un rango óptimo, mientras que en los demás elementos y relaciones se presentó desbalance (Berstch 1987).

\footnotetext{
${ }^{4}$ Argel, P. 1998. Fertilización de Cratylia argentea. (entrevista). San José, CR. CIAT. Comunicación personal.
} 
Cuadro 1. Características químicas del suelo antes de la siembra. Puriscal, Costa Rica. 1998.

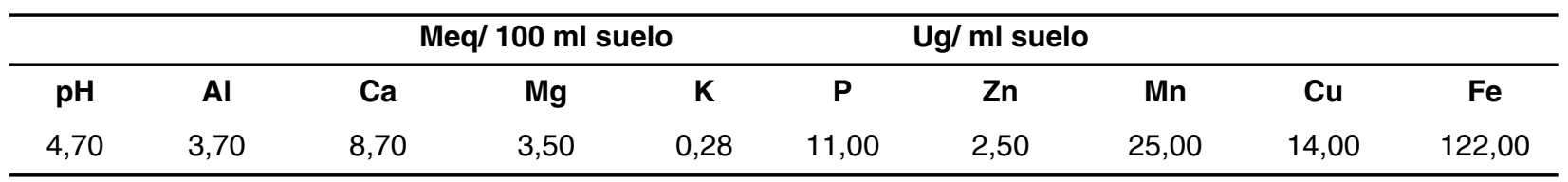

Fuente: Laboratorio de suelos, foliares y aguas del INTA.

El Cuadro 2 presenta los eventos que se identificaron durante el periodo de establecimiento, desarrollo, cosecha y primera poda del arbusto. Se observó que la emergencia de plántulas es muy rápida o sea las semillas tienen muy baja latencia, lo cual coincide con lo mencionado por (Argel et al. 2002). Sin embargo, el crecimiento de los arbustos durante el primer año es lento; durante el lapso de los primeros dos meses, el crecimiento diario fue de aproximadamente 0,12 cm por día, por esta razón, se debe tener la plantación libre de malezas que no retrasen el desarrollo del cultivo. La primera floración se logró en el mes de setiembre, o sea, 12 meses después de la siembra. La primera cosecha se dio a los 4,93 meses después de la floración. La floración se mantuvo durante todo el periodo de cosecha, con una disminución paulatina hacia el final del ciclo. La poda, durante el primer año, se realizó al inicio de las lluvias o sea en abril, inmediatamente después de terminada la primera cosecha. Sin embargo, durante los años siguientes, se realizó en cada planta al momento de terminar la cosecha, a una altura de $50 \mathrm{~cm}$.

Cuadro 2. Información recopilada de la siembra a la primera poda. Puriscal, Costa Rica. 2000.

\begin{tabular}{ll}
\hline Evento & Características \\
\hline Siembra & Setiembre, 1998 \\
Emergencia de las plantas, días & 10 \\
Altura promedio de la plantación a los 60 días, cm & 7,30 \\
Tasa de crecimiento inicial, cm/ día & 0,12 \\
Periodo de floración & Setiembre 1999 - marzo 2000 \\
Altura al inicio de la floración, cm & 210 \\
Tasa de crecimiento durante el periodo (210 días), cm/ día & 0,70 \\
Periodo de cosecha & Febrero - marzo, 2000 \\
Periodo de poda & Primera semana de abril, 2000 \\
Altura de poda, cm & 50 \\
\hline
\end{tabular}

El Cuadro 3 muestra que el inicio de la floración durante los siete años estudiados se presentó durante el mes de setiembre, sin embargo, la floración es poco sincronizada, se mantuvo durante tres meses, de modo que generalmente al terminar la cosecha en abril todavía prevalecen alguna inflorescencia, la cual no se desarrolla ya que el exceso de humedad las deteriora y luego se desprenden. Información similar a la reportada por Argel et al. (2002) y Lascano et al. (2002) 
Cuadro 3. Información recopilada a partir del segundo año de cosecha hasta el final del estudio. Puriscal, Costa Rica. 2007.

\begin{tabular}{ll}
\hline Evento & Característica \\
\hline Inicio de floración & Setiembre \\
Final de la floración & Abril \\
Color de la flor & $98,80 \%$ de plantas color lila y $1,20 \%$ de \\
& plantas color blanco \\
Pérdida de flores & Las que emergen al final de la cosecha \\
Tipo de fruto & Legumbre dehiscente \\
Cosecha & Febrero, marzo y abril \\
Tamaño promedio de vainas, cm & 13,80 \\
Cantidad promedio de semillas por vaina & 7,70 \\
Germinación de la semilla producida, \% & 85 - 90 \\
Poda de la plantación & Al terminar la cosecha cada año \\
Semillas por kilogramo & $3.650,00$ \\
Presencia de hongos en la semilla & Penicillium sp y Aspergillus sp \\
Pérdida de plantas durante el quinto año, \% & 3,00 \\
\hline
\end{tabular}

Se realizó la cosecha para cada periodo entre febrero y abril, efectuándose diariamente de forma manual, cosechando las vainas que iban cambiando de coloración verde a café claro. Las vainas después de cosechadas se terminan de secar al sol en lonas cubiertas con una malla para evitar la pérdida de semillas por el fenómeno de dehiscencia que presenta este cultivo, ya que las valvas se abren fácilmente y expulsan la semilla.

Argel et al. (2002) mencionan que la semilla debe ser almacenada en refrigeración en bolsas de papel, plástico o polietileno. Además, recomiendan que las semillas que van a ser guardadas por más de un año tratarlas con insecticida para evitar el ataque de insectos de granos almacenados.

Cada año se realizó la prueba de germinación, la cual demostró que la semilla producida es de muy buena calidad presentando de 85 a $95 \%$ de germinación; la cual disminuye para el año siguiente de 5 a $10 \%$ si se mantiene a una temperatura aproximada de $13 \stackrel{\circ}{\circ}$. Sin embargo, la germinación disminuye en un $50 \%$ al año siguiente si se mantiene a temperatura ambiente (datos similares a los reportados por Argel et al. 2002).

Durante el quinto año de producción hubo pérdida del $3 \%$ de las plantas. El análisis de laboratorio indicó presencia de los hongos Nectria spp (cáncer en el tallo), Phytophthora sp. y Graphium spp (pudrición del tallo) (Laboratorio de Fitoprotección de Cultivos, INTA 2004)

La producción de semilla a partir del segundo año (Cuadro 4), fue superior a la reportada por Argel et al. 2001, quienes reportan producciones de 500 a $700 \mathrm{~kg}$ de semilla/ ha con plantas cortadas a $30 \mathrm{~cm}$ de altura y fertilizadas con fósforo al inicio de la época lluviosa en Atenas, Costa Rica. 
Cuadro 4. Producción de semilla de Cratylia argentea durante siete años. Puriscal, Costa Rica. 2007.

\begin{tabular}{cc}
\hline Año & Producción de semilla, $\mathbf{t}$ ha \\
\hline 1 & 0,12 \\
2 & 0,60 \\
3 & 2,02 \\
4 & 2,45 \\
5 & 1,89 \\
6 & 1,36 \\
7 & 0,83 \\
\hline
\end{tabular}

La semilla en la mayoría de los años evaluados tuvo presencia de los hongos Penicillium $s p$ y Aspergillus sp (Laboratorio de Fitoprotección de Cultivos, INTA 2004).

Se puede observar que la producción de semilla durante siete años se comportó de forma parabólica, presentando la máxima producción a los cuatro años de edad de las plantas. Luego empezó a disminuir de modo que al sétimo año la producción es similar a la del segundo año (Figura 1). El modelo de regresión que describe este efecto es el siguiente: $Y=-1,58+1,72 x-0,20 x^{2}$.

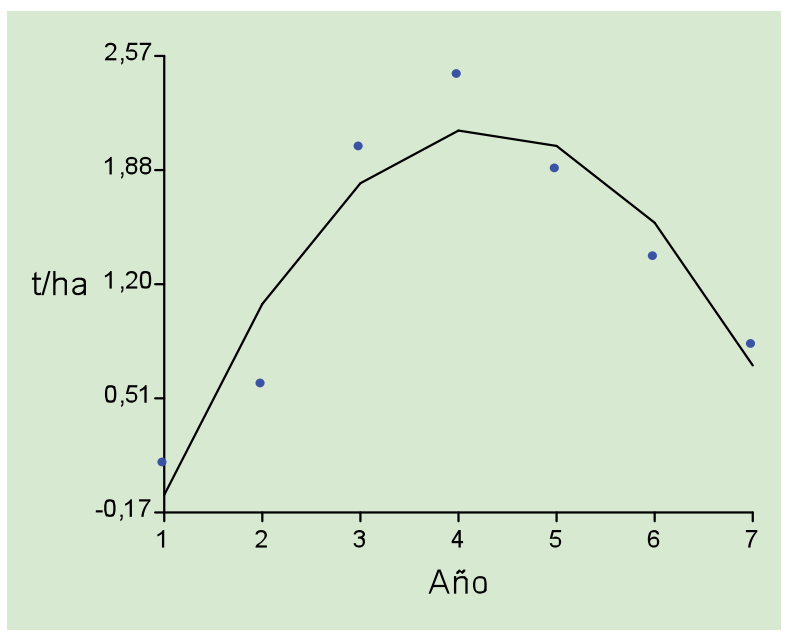

Figura 1. Producción de semilla de Cratylia argentea. Puriscal, Costa Rica. 2007.

\section{CONCLUSIONESY RECOMENDACIONES}

Teniendo en consideración las condiciones bajo las cuales se llevó a cabo el presente estudio, se formularon las siguientes conclusiones y recomendaciones:

Durante el establecimiento del cultivo de Cratylia argentea se debe tener un estricto control de malezas ya que el crecimiento es muy lento.

La floración del arbusto se prolonga desde setiembre hasta abril.

Las flores que emergen al final del periodo no se desarrollan ya que el exceso de humedad hace que los botones florales se deterioren y se desprendan.

La producción de semilla durante siete años de estudio se comporta de forma parabólica, presentándose la máxima producción a los cuatro años de edad de las plantas.

La cosecha debe realizarse de febrero a abril, continuamente, preferiblemente todos los días para evitar pérdidas por dehiscencia.

Si hay presencia de hongos en las semillas, se debe tratar con un fungicida sistémico protector.

Se recomienda el tratamiento de la semilla con un fungicida sistémico protector, ya que hubo presencia de hongos en la misma. En el reporte del laboratorio se recomienda el fungicida Carboxin + Captan.

\section{AGRADECIMIENTO}

Los autores expresan su agradecimiento al señor Edén Delgado Delgado funcionario del Ministerio de Agricultura y Ganadería de la Región Central Sur, al Dr. Pedro Argel Montalvo de la oficina de CIAT en Costa Rica y a los ingenieros Marco Vinicio Castro Bonilla y Beatriz Sandoval Carvajal del INTA por su valiosa colaboración en el desarrollo del presente estudio. 


\section{LITERATURA CITADA}

Argel, P.; Hidalgo, C.; González, J.; Lobo, M.; Acuña, V.; Jiménez, C. 2001. Cultivar Veraniega. s.e. (Boletín Técnico). San José, CR, $22 \mathrm{p}$.

Giraldo, G.; Peters, M.; Lascano,C. 2002. Producción artesanal de semillas de Cratylia (Cratylia argentea). s.e. (Boletín técnico). San José, CR, 16 p.

Bernal, J. 1991. Pastos y forrajes tropicales. $2^{\text {a }}$ ed. Colombia. Banco ganadero. p. 273.

Bertsch, F. 1987. Manual para interpretar la fertilidad de los suelos de Costa Rica. Costa Rica. $2^{\text {a }}$ ed. San José, Costa Rica, Universidad de Costa Rica. 82 p.

INTA (Instituto Nacional de Innovación y Transferencia en Tecnología Agropecuaria, CR). 2004. Reporte del Laboratorio de Fitoprotección de Cultivos. 1 p.

Lascano, C.; Ávila P.; Bueno, G.; Argel, P.; 2002. Cultivar Veranera (Cratylia argentea Desvaux O. Kuntze). (en línea). Bogotá, Co. CIAT. Consultado 10 de dic 2008. Disponible en http://www.ciat.cgiar. org/forrajes/pdf/cratylia_argentea_cv_ veranera. $24 \mathrm{p}$.

Martínez, A. 1992. Disponibilidad, composición botánica, selectividad y calidad nutritiva de seis asociaciones gramínea leguminosa. Tesis, Mag Sc., CATIE. Turrialba, Costa Rica. 120 p.

Orozco, E. 2005. Bancos forrajeros. s.e. (Boletín Técnico). San José, Costa Rica, $47 \mathrm{p}$.

2003. El arbusto forrajero Cratylia argentea cultivar Veraniega. s.e. (Boletín Técnico) San José, Costa Rica, 23 p.
UNC (Universidad Nacional de Córdoba, AR). 2001. Info Stat: Sofware de Estadística y Biometría Info Stat 2001. Versión 1.0. 
ALCANCES TECNOLÓGICOS, AÑO 6, NÚMERO 1 